\title{
Utilização da pressão positiva contínua nas vias aéreas (CPAP) durante atividade física em esteira ergométrica em portadores de doença pulmonar obstrutiva crônica (DPOC): comparação com o uso de oxigênio*
}

\author{
Flávio danilo Mungo Pissulin ${ }^{1}$, Alcides Guimarães², Lúcio Benedicto Kroll ${ }^{3}$, Michel J orge CeCílio ${ }^{1}$
}

Objetivo: Analisar os efeitos da pressão positiva contínua nas vias aéreas (CPAP) sobre os parâmetros espirométricos, capacidade vital forçada (CVF) e volume expiratório forçado no primeiro segundo $\left(V F_{1}\right)$, freqüência respiratória (f), saturação de $\mathrm{O}_{2}\left(\mathrm{SaO}_{2}\right)$, freqüência cardíaca (fc), pressão arterial

sistólica (PAS) e diastólica (PAD). Materiais e métodos: Nove indivíduos do sexo masculino, portadores de DPOC, foram submetidos a atividade física em esteira ergométrica utilizando-se a CPAP com $\mathrm{FiO}_{2}$ a $30 \%$ e em ar comprimido, sendo verificada a CVF e $0 \mathrm{VEF}_{1}$ ao repouso e após o término da atividade física e a f, a $\mathrm{SaO}_{2}$, a fc, a PAS e PAD ao repouso e durante a atividade física. Resultados:

Com a aplicação da CPAP com $\mathrm{FiO}_{2}$ a $30 \%$ e em ar comprimido as médias da CVF foram significativamente maiores $(2,13 \pm 0,38 \times 2,27 \pm 0,52$ e $1,90 \pm 0,51 \times 2,10 \pm 0,46$, respectivamente), $0 \mathrm{VEF}_{1}$ aumentou $(0,60 \pm 0,21 \times 0,90 \pm 0,33)$ ao aplicar a CPAP em ar comprimido, as médias da $\mathrm{f}$ e da fc foram significativamente menores $(\alpha=0,05)$ nas duas situações estudadas e a média da $\mathrm{SaO}_{2}$ foi significativamente maior ao usar a CPAP com FiO a $30 \%(\alpha=0,05)$. Conclusão: Ao utilizar a CPAP, nas duas situações estudadas, a CVF aumentou, de maneira transitória, o que ocorreu com $0 \mathrm{VEF}_{1}$ somente com a aplicação da CPAP em ar comprimido, a f e a fc diminuíram e a $\mathrm{SaO}_{2}$ apresentou maior média ao ser aplicada a CPAP Com FiO 2 a 30\%. (J Pneumol 2002;28(3):131-136)

Use of continuous positive airway pressure (CPAP) during physical activities on an ergometric treadmill performed by individuals with chronic obstructive pulmonary disease (COPD): comparison with the use of oxygen

\begin{abstract}
Objective: To analyze the effects of the continuous positive airway pressure (CPAP), with $30 \%$ of $\mathrm{FiO}_{2}$ and in compressed air, used during the performance of submaximal physical activity on an ergometric treadmill. Materials and methods: Nine male individuals with COPD were submitted to physical activity on the ergometric treadmill using CPAP with $30 \% \mathrm{FiO}_{2}$. The authors evaluated the behavior of the spirometric parameters, the forced vital capacity (FVC), the forced expiratory volume in the first second $\left(\mathrm{FEV}_{1}\right)$ before and after physical activity, the respiratory frequency (f), the arterial oxygen saturation $\left(\mathrm{SaO}_{2}\right)$, the cardiac frequency (cf), the systolic arterial pressure (SAP), and the diastolic arterial pressure (DAP) at rest and during physical activity. Results and conclusion: Results have shown that, when using CPAP in both situations analyzed, there has been an increase in the FVC and a decrease in $f$ and in of with statistically significant difference $(\alpha=0.05)$. $\mathrm{SaO}_{2}$ has showned a significantly higher mean when CPAP was used with $30 \%$ of $\mathrm{FiO}_{2}$ $(\alpha=0.05)$. The FEV ${ }_{1}$, the SAP and DAP have maintained the same value in the statistical analyses.
\end{abstract}

* Trabalho realizado no setor de Fisioterapia em Pneumologia e Cardiologia do Hospital Universitário “Dr. Domingos Leonardo CerávoIo", Presidente Prudente, SP. Órgão Financiador: Programa de Capacitação de Docentes da Unoeste - PROBIC.

1. Professor de Fisioterapia Respiratória e Cardiovascular.

2. Professor Titular de Fisiologia e Biofísica.

3. Professor de Bioestatística.
Endereço para correspondência - Rua Benedito Garcia Borges, 123 19025-140 - Presidente Prudente, SP. Tel. (18) 221-1082.

Recebido para publicação em 20/6/01. Aprovado, após revisão, em 18/3/02. 
Descritores - Teste de esforço. Volume expiratório forçado. Capacidade vital. Espirometria. Freqüência cardíaca. Pneumopatias obstrutivas. Respiração com pressão positiva.

Key words - Exercise test. Forced expiratory volume. Vital capacity. Spirometry. Heart rate. Obstructive lung diseases. Positive-pressure respiration.

\section{INTRODUÇÃO}

A dispnéia é o maior desafio para um indivíduo portador de doença pulmonar obstrutiva crônica (DPOC), durante a realização de suas atividades de vida diária. Alguns fatores são responsáveis por esse déficit respiratório, entre eles, a destruição parcial do tecido pulmonar, que acarreta diminuição da área de troca gasosa, o colapso precoce das vias aéreas e o aumento da complacência pulmonar, levando a alterações no funcionamento dos músculos respiratórios, o que eleva o custo da respiração(1).

Os problemas existentes ao repouso agravam-se durante a realização de atividade física. A necessidade do aumento da freqüência respiratória para potencializar a ventilação alveolar e aumentar a captação de oxigênio $\left(\mathrm{O}_{2}\right)$ gera, em indivíduos com DPOC, hiperinsuflação dinâmica(2-4).

A reabilitação pulmonar, nesses casos, não visa a meIhora direta nos níveis da função pulmonar, mas a diminuição da dispnéia e melhor capacidade para a realização de exercícios, através da possibilidade da mudança nas propriedades bioquímico-fisiológicas das fibras musculares $^{(5)}$. Pacientes com DPOC grave apresentam grande perda na função pulmonar e, como conseqüência, incapacidade física grave, com dificuldade para a realização de treinamento físico.

A pressão positiva contínua nas vias aéreas (CPAP) exerce ação sobre o fechamento precoce das vias aéreas e sobre o trabalho muscular respiratório em indivíduos portadores de DPOC em insuficiência respiratória aguda(6-8).

Este estudo visa avaliar aspectos fisiopatológicos do uso da CPAP em portadores de DPOC sobre os parâmetros espirométricos, freqüência respiratória (f), saturação arterial de oxigênio $\left(\mathrm{SaO}_{2}\right)$, freqüência cardíaca (fc), pressão arterial sistólica (PAS) e diastólica (PAD), antes e após os portadores de DPOC serem submetidos à atividade física em esteira ergométrica.

\section{Material e mÉtodos}

Para a realização do presente trabalho, foram selecionados nove indivíduos do sexo masculino, com idade média de 69,7 anos, todos portadores de doença pulmonar obstrutiva crônica, ex-tabagistas, apresentando os seguin-
Siglas e abreviaturas utilizadas neste trabalho

bpm - Batimento por minuto

$\mathrm{CmH}_{2} \mathrm{O}$ - Centímetros de água

$\mathrm{CO}_{2}$ - Dióxido de carbono

CPAP - Pressão positiva contínua nas vias aéreas

CVF - Capacidade vital forçada

DPOC - Doença pulmonar obstrutiva crônica

$f$ - Freqüência respiratória

fc - Freqüência cardíaca

$\mathrm{FiO}_{2}$ - Fração inspirada de oxigênio

ipm - Incursões por minuto

$\mathrm{mlO}_{2} / I$ vent. - Mililitros de oxigênio por litro de ventilação

$\mathrm{mmol} / \mathrm{l}$ - Milimol por litro

$\mathrm{O}_{2}$ - Oxigênio

PAD - Pressão arterial diastólica

PAS - Pressão arterial sistólica

PEEP - Pressão positiva expiratória final

PEEPi - Pressão positiva expiratória final intrínseca

PIP - Ponto de igual pressão

$\mathrm{SaO}_{2}$ - Saturação arterial de oxigênio

$V E F_{1}$ - Volume expiratório forçado no primeiro segundo

VNI - Ventilação não invasiva

tes valores individuais de $\mathrm{VEF}_{1}: 0,70,0,37,0,83,0,41$, $0,79,1,03,0,87,0,81,1,66$, com média de $0,83 \mathrm{~L}$ $( \pm 0,38 \mathrm{~L})$, verificados anteriormente à realização do primeiro teste. Após as explicações necessárias, os indivíduos assinaram termo de consentimento para participar da pesquisa, que somente foi realizada após parecer favorável emitido pelo Comitê de Ética em Pesquisa da Unoeste.

No setor de reabilitação pulmonar do Hospital Universitário "Dr. Domingos Leonardo Cerávolo", foram realizados, em cada indivíduo, os seguintes procedimentos:

- solicitou-se que se mantivessem em repouso, sentados, por 10 minutos;

- foi verificada a fc através do método palpatório da artéria radial;

- verificou-se a f, através da observação da caixa torácica;

- em seguida, observaram-se a PAS e PAD por método não-invasivo, auscultatório, utilizando-se estetoscópio (Littmann, modelo Cardiology II SE) e esfigmomanômetro (Labtton);

- $\mathrm{S} \mathrm{SaO}_{2}$ foi verificada através de método não invasivo, utilizando-se a oximetria de pulso (Criticare modelo 504);

- a prova de função pulmonar foi realizada em espirômetro VT $130 \mathrm{SL}$, acoplado ao microcomputador, com programa Spiromatic 4.0, com o paciente em posição ortostática, sendo solicitada inspiração máxima e, posteriormente, expiração máxima forçada, para obtenção dos parâmetros espirométricos e determinação do tipo e grau de distúrbio ventilatório, segundo Pereira et al.(14) e, conseqüentemente, os valores da CVF e do $V_{E F}$, para posterior análise. 
Para a realização da atividade física, foi calculada a freqüência cardíaca de treinamento (FCt), através da fórmula descrita por Karvonen et al.(15), sendo que a percentagem de trabalho foi fixada em $60 \%$ para todos os indivíduos.

Em seguida, os pacientes foram conduzidos até a esteira ergométrica (Unisen, modelo Star Trac), regulada a uma velocidade e inclinação para que se atingisse a freqüência cardíaca de treinamento, mantendo-se por no máximo 15 minutos, sendo que, na presença de sinais e sintomas de descompensação ou fadiga, a atividade física era interrompida. A velocidade e a inclinação necessárias para obter a FCt e, como conseqüência, manter a mesma atividade física submáxima foram as mesmas na situação sem aplicação da CPAP, CPAP $\mathrm{COm} \mathrm{FiO}_{2}$ a $30 \%$ e CPAP em ar comprimido.

Durante a atividade física, na esteira ergométrica, foram verificadas a fc, PAS e PAD, a $\mathrm{f} \mathrm{a} \mathrm{SaO}_{2}$, nos tempos de $3,6,9,12$ e 15 minutos, porém, quando era necessária a interrupção da atividade, anotavam-se os dados até o momento da intervenção.

Ao término de cada sessão de atividade física na esteira era realizada uma nova prova de função pulmonar, idêntica à anterior.

A pós uma semana da realização desses procedimentos experimentais, os indivíduos retornaram e foram submetidos à mesma seqüência anterior, porém, durante a atividade física, na esteira ergométrica, foi aplicada CPAP, de fluxo contínuo, acoplado a fluxômetro de oxigênio $\left(\mathrm{O}_{2}\right)$, utilizando-se fração inspirada de $\mathrm{O}_{2}\left(\mathrm{FiO}_{2}\right)$ a $30 \%$, via máscara fascial, com válvula de pressão positiva expiratória final (PEEP), entre 4 e $6 \mathrm{cmH}_{2} \mathrm{O}$. Posteriormente, aplicouse CPAP com as mesmas características, porém, sem a utilização de $\mathrm{FiO}_{2}$ de $30 \%$, ou seja, utilizou-se CPAP a $21 \%$ de $\mathrm{FiO}_{2}$ (em ar comprimido).

Para a análise dos valores da CVF e $\mathrm{VEF}_{1}$, sem e com CPAP, em situação pré e pós-atividade física e para o tempo de atividade física foi utilizado o teste t de Student, aplicado para pares combinados, conforme o descrito em Hoel ${ }^{(16)}$.

As observações da f, $\mathrm{SaO}_{2}$, fc, PAS e PAD, em função do tempo de uso da esteira (repouso, 3', 6', 9', 12', 15'), foram analisadas estatisticamente por um delineamento inteiramente casualizado, num esquema fatorial com dois fatores (A: sem uso e com uso de CPAP; B: tempo de uso da esteira, ou seja, repouso, $\left.3^{\prime}, 6^{\prime}, 9^{\prime}, 12^{\prime}, 15^{\prime}\right)$, totalizando 12 tratamentos.

\section{Resultados}

Verificou-se que a comparação entre as médias da CVF (Figura 1), pré e pós-atividade física, sem a aplicação de CPAP, produziu um valor de tigual a 0,15 , o que permite afirmar que a diferença que ocorreu entre as respectivas médias não foi estatisticamente significativa, porém, a comparação entre as médias da CVF pré e pós, com a aplicação da CPAP com $\mathrm{FiO}_{2}$ de $30 \%$, produziu um valor de $t$ igual a $-2,25$, mostrando que a diferença entre as médias foi estatisticamente significativa, o que também ocorreu quando da aplicação da CPAP em ar comprimido $(\mathrm{t}=-1,39)$.

Com relação ao $\mathrm{VEF}_{1}$ (Figura 2), não houve diferença estatisticamente significativa ao realizar atividade física sem a aplicação da CPAP $(t=-1,5)$ e ao aplicar CPAP Com $\mathrm{FiO}_{2}$ a $30 \%(t=-1,5)$. Porém, a aplicação da CPAP com ar comprimido produziu diferença significativa $(t=-4,17)$.

Como mostra a Figura 3, as médias da f, ao repouso, demonstram valores estatisticamente semelhantes, ao passo que aos 3, 6, 9, 12 e 15 minutos de atividade física as médias foram significativamente inferiores quando se utilizou CPAP com $\mathrm{FiO}_{2}$ a $30 \%$ e CPAP com ar comprimido $(\alpha=0,05)$. Pode-se observar a tendência a taquipnéia

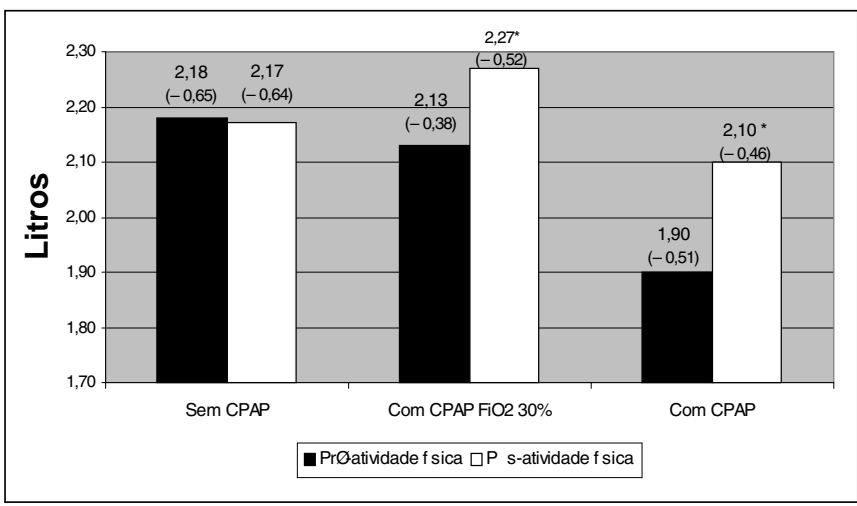

Figura 1 - Demonstração da média dos valores da capacidade vital forçada (CVF), em litros, pré e pós-atividade física, sem CPAP, com CPAP FiO $230 \%$ e com CPAP, nos sujeitos estudados $(\alpha=0,05)$

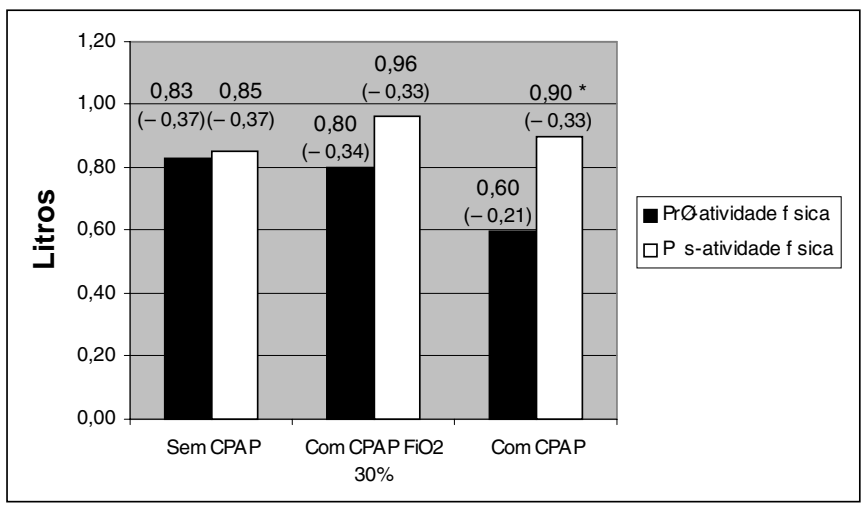

Figura 2 - Demonstração das médias dos valores do volume expiratório forçado no primeiro segundo $\left(V E F_{1}\right)$, em litros, pré e pósatividade física, sem CPAP, com CPAP FiO $30 \%$ e com CPAP, nos sujeitos estudados $\left({ }^{*} \alpha=0,05\right)$ 


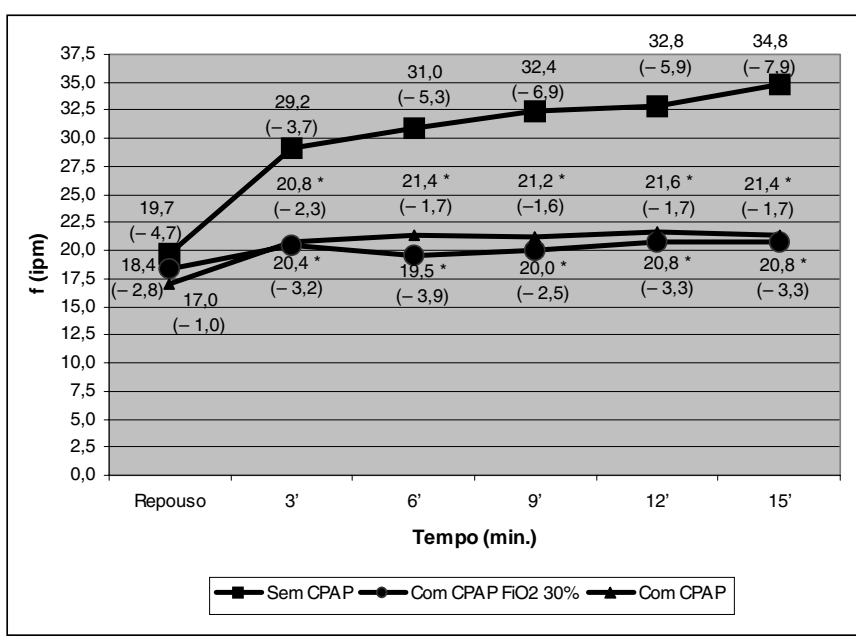

Figura 3 - Demonstração das médias dos valores da freqüência respiratória $(f)$, em respirações por minuto (rpm), sem CPAP, com CPAP FiO $230 \%$ e com CPAP, nos sujeitos estudados $(\alpha=0,05)$

quando não foi utilizada a CPAP e, ao contrário, estabilidade nas médias da f quando do uso da CPAP nas duas situações estudadas.

A comparação entre as médias da $\mathrm{SaO}_{2}$ (Figura 4) demonstrou diferença estatisticamente significativa, sendo que 0 uso da CPAP Com $\mathrm{FiO}_{2}$ a $30 \%$ produziu a maior média, $98,1 \%$, contra $94,5 \%$ sem a aplicação da CPAP e $92,4 \%$ quando se utilizou CPAP em ar comprimido $(\alpha=0,05)$. A comparação entre as médias ao repouso, $3,6,9,12$ e 15 minutos demonstrou semelhança entre as três situações analisadas.

A comparação entre as médias da fc (Figura 5), durante atividade física em esteira, mostrou diferença significativa, com média de $98,7 \mathrm{bpm}$, quando se utilizou CPAP com ar comprimido, seguido de $104,7 \mathrm{bpm}$ com CPAP e $\mathrm{FiO}_{2}$ a $30 \%$ e $111,8 \mathrm{bpm}$, quando a atividade física foi realizada sem CPAP. As médias, ao repouso e entre os tempos de atividade física, foram semelhantes. Quando comparado o repouso com os tempos de atividade física (3, $6,9,12$ e 15 minutos), observam-se valores de fc estatisticamente menores ao repouso nas três situações estudadas $(\alpha=0,05)$.

Com relação à PAS e PAD, foram observados valores estatisticamente semelhantes nas três situações estudadas.

\section{DISCUSSÃO}

A dificuldade encontrada para a realização de treinamento físico em indivíduos portadores de DPOC cria uma expectativa em buscar alternativas que, efetivamente, contribuam para melhorar o desempenho durante os treinamentos e, possivelmente, melhorar a qualidade de vida desses pneumopatas.

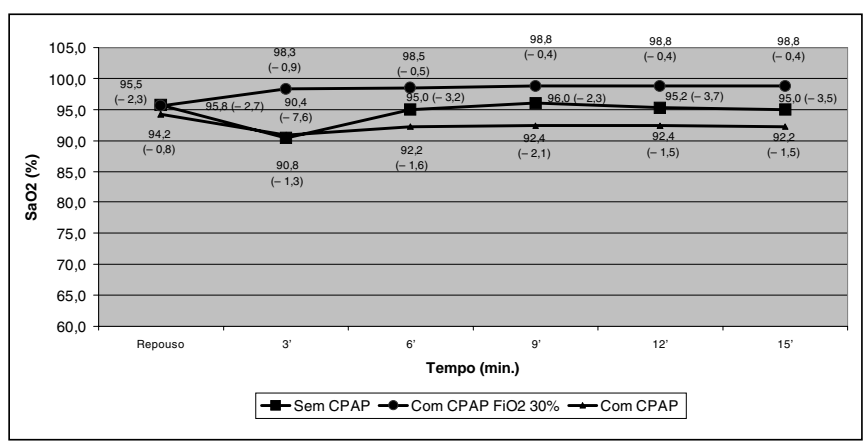

Figura 4 - Demonstração das médias dos valores da saturação arterial de $\mathrm{O}_{2}\left(\mathrm{SaO}_{2}\right)$, em percentagem (\%), sem CPAP, com CPAP FiO $30 \%$ e com CPAP, nos sujeitos estudados

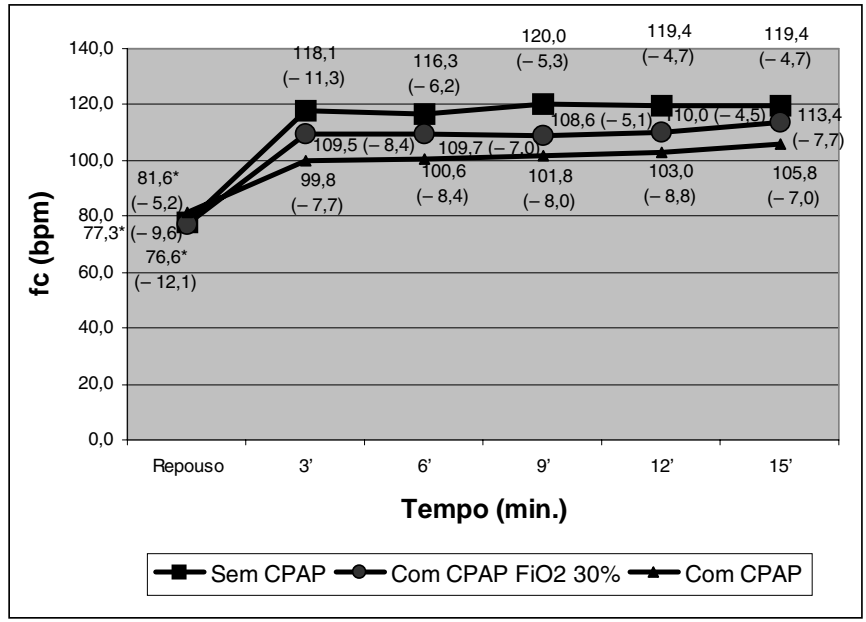

Figura 5 - Demonstração das médias dos valores da freqüência cardíaca em batimentos por minuto (bpm), sem CPAP, com CPAP $\mathrm{FiO}_{2} 30 \%$ e com CPAP, nos sujeitos estudados ( $\left.{ }^{*} \alpha=0,05\right)$

A literatura tem demonstrado que a utilização da oxigenioterapia com cateter nasal pode auxiliar na realização de exercícios físicos, em indivíduos portadores de DPOC que apresentam níveis de $\mathrm{SaO}_{2} \leq 90 \%{ }^{(18)}$. Porém, na prática clínica, tem-se observado que, em muitas situações, a utilização da mesma traz desconforto e dificuldade para atingir a freqüência cardíaca de treinamento, necessária para que os efeitos da atividade física aeróbica sejam obtidos $^{(9,10)}$. Dessa forma, vários estudos ${ }^{(11-13,17)}$ vêm sendo realizados para observar os efeitos das técnicas de ventilação não-invasiva nessas situações.

$\mathrm{Na}$ análise dos índices obtidos nas provas de função pulmonar, em particular a CVF obtida 15 minutos após 0 término da atividade física (CVF pós-atividade física) nas duas situações estudadas e comparada com a fase préatividade física, houve maior diferença entre os valores, 0 que não ocorreu quando a atividade física foi realizada na ausência da CPAP. A pesar de 0 aumento da quantidade de ar expirado ter sido estatisticamente significativo, não se 
pode cometer o erro de pensar que isso tenha significância de prova broncodilatadora positiva, até porque não foi utilizado nenhum tipo de fármaco; mas é razoável a idéia de que a melhora da quantidade de ar exalado possa ter contribuído para a realização do exercício físico, uma vez que os níveis da f foram significativamente menores. É importante relembrar a equação do volume minuto pulmonar, que é obtido a partir do produto do volume corrente pela freqüência respiratória. A hiper-reatividade das vias aéreas, que eleva a resistência das mesmas, é um problema que pode acontecer durante a prática de exercícios ${ }^{(18,20)}$. Essa ocorrência, comum a indivíduos portadores de DPOC, provavelmente foi evitada pela aplicação da PEEP, que estabiliza as vias aéreas, evitando colapsos ${ }^{(8)}$. 0 'Donnel et al.(11) observaram, de maneira semelhante, a melhora do volume expiratório ao aplicarem CPAP, durante atividade física em bicicleta ergométrica, com pressão entre 4 e $5 \mathrm{CmH}_{2} \mathrm{O}$.

$O V F_{1}$, que analisa o volume de ar exalado em um segundo, durante a manobra da CVF, não apresentou 0 mesmo comportamento estatístico que a CVF, permanecendo sem diferença significativa, sem e com a aplicação da CPAP com $\mathrm{FiO}_{2}$ a $30 \%$, sendo que, em ar comprimido, a média de $\mathrm{VEF}_{1}$ aumentou significativamente após a atividade física. Porém, quando se utilizou a CPAP Com $\mathrm{FiO}_{2}$ a $30 \%$, observou-se aumento mais evidente do $\mathrm{VEF}_{1}$ quando comparado com a situação sem CPAP (Figura 2). As possibilidades aventadas durante a discussão da CVF podem também ter sido as causas do aumento do $\mathrm{VEF}_{1}$ quando se utilizou a CPAP.

Devemos ressaltar que o estado pré-atividade física referente aos parâmetros espirométricos $\mathrm{CVF}_{\text {e }} \mathrm{VEF}_{1}$ (Figuras 1 e 2) apresentou valores médios menores quando da aplicação da CPAP nas duas situações estudadas, provavelmente por maior resistência das vias aéreas e, mesmo assim, os indivíduos conseguiram realizar a atividade física com a mesma intensidade de esforço e com menor f e fc. Pode-se observar que ocorreu maior diferença entre as médias pré e pós-atividade física, tanto da CVF como de $\mathrm{VEF}_{1}$, ao utilizar a CPAP, apesar da insignificância estatística da diferença das médias de $\mathrm{VEF}_{1}$ quando se aplicou a CPAP com $\mathrm{FiO}_{2}$ a $30 \%$ (Figura 2).

0 aumento da f durante a realização de atividade física pode diminuir o tempo expiratório, ocorrendo a necessidade de utilizar os músculos expiratórios na tentativa de vencer 0 aumento da resistência nas vias aéreas em portadores de DPOC. Porém, esse mecanismo fisiológico, que busca a manutenção de bom volume corrente, esbarra na presença do colapso precoce das vias aéreas, adiantando o ponto de igual pressão para vias aéreas de menor calibre, levando à instalação da hiperinsuflação pulmonar ${ }^{(1,2,4)}$. Neste estudo, tanto a aplicação da CPAP com $\mathrm{FiO}_{2}$ de $30 \%$, como em ar comprimido, gerou níveis de f estatistica- mente menores $(\alpha=0,05)$, quando comparados com o controle (Figura 3). Isso pode refletir a interferência da PEEP sobre o ponto de igual pressão, retardando o fechamento precoce das vias aéreas, mantendo um bom volume corrente e impedindo a formação da hiperinsuflação dinâmica. Petrof et al.(12) não observaram diferenças significantes em relação a f. Provavelmente, o uso da PEEP entre 7,5 e $10 \mathrm{CmH}_{2} \mathrm{O}$ foi excessivo e não evitou a hiperinsuflação pulmonar, o que ficou demonstrado pelo mesmo estudo, haja vista que o trabalho dos músculos abdominais aumentou durante a aplicação da CPAP. 0 mesmo foi demonstrado por Bianchi et al.(17) que, apesar de usarem PEEP de $6 \mathrm{CmH}_{2} \mathrm{O}$, utilizaram a bicicleta como ergômetro. $\mathrm{Na}$ posição sentada, os níveis de CRF são mais baixos ${ }^{(19)}$ e, por isso, os níveis de PEEP ideal devem ser menores ainda.

A comparação entre as médias da $\mathrm{SaO}_{2}$, que produziu maiores níveis ao utilizar CPAP com $\mathrm{FiO}_{2}$ a $30 \%(98,1 \%)$, provavelmente ocorreu devido à utilização de níveis de oxigênio supra-atmosféricos, uma vez que, ao utilizar CPAP em ar comprimido, não foi possível manter os mesmos níveis $(92,4 \%)$, sendo que a realização da atividade física sem a aplicação de CPAP obteve média de $94,5 \%$. A ação da PEEP pode não ter interferido sobre a quantidade de ar inspirado fresco, disponível para a troca gasosa (ventilação alveolar)(1), mas, provavelmente, alterou o volume total de ar que deixa o pulmão a cada minuto(1), proporcionando maior exalação de $\mathrm{CO}_{2}$, evitando seu acúmulo nos alvéolos e conseqüente aumento em nível sistêmico, o que geraria acúmulo de lactato e fadiga muscular. Essa afirmação baseia-se no fato de que, durante a atividade física, sem a utilização da CPAP, a mesma foi interrompida em três indivíduos, na fase de déficit de $\mathrm{O}_{2}$, por fadiga, ao passo que, com a utilização da CPAP, nas duas situações estudadas, isso não ocorreu, prolongando-se o tempo de atividade física.

A utilização de $\mathrm{O}_{2}$ durante atividade física é preconizada quando se têm níveis de $\mathrm{SaO}_{2} \leq 90 \%{ }^{(18)}$. Porém, a sensação de dispnéia é variável e pode interferir sobre a realização da atividade física. O'Donnell et al.(11) não analisaram a $\mathrm{SaO}_{2}$, ao aplicar CPAP com pressões entre 4 e $6 \mathrm{cmH}_{2} \mathrm{O}$, durante atividade física em bicicleta ergométrica, porém, utilizaram $\mathrm{FiO}_{2}$ de $30 \%$ e verificaram que a sensação de dispnéia diminuiu significativamente, ao empregar CPAP.

A atividade física, em indivíduos portadores de DPOC, pode promover hiperinsuflação dinâmica e, como conseqüência, aumento da pressão intratorácica e do volume alveolar, aumento da resistência vascular pulmonar, diminuição do retorno venoso, bem como compressão direta do coração, pela expansão pulmonar, alterando a complacência ventricular ${ }^{(4,7)}$. Uma das formas de compensação, para a manutenção de bom débito cardíaco, é o au- 
mento da freqüência cardíaca. Dessa forma, tanto a aplicação da CPAP com $\mathrm{FiO}_{2}$ a 30\%, como a CPAP com ar comprimido podem ter contribuído para evitar a hiperinsuflação dinâmica, e, como conseqüência, obter níveis de fc significativamente menores do que quando não se utilizou CPAP. Petrof et al.(12) não encontraram diferença significativa quanto à $\mathrm{fc}$. Provavelmente, $\mathrm{o}$ fato de esses autores terem utilizado níveis de PEEP entre 7,5 e $10 \mathrm{cmH}_{2} \mathrm{O}$ e a bicicleta como ergômetro, que pela postura sentada mantém menores valores de capacidade residual funcional(21), supra-estimou o valor da PEEP ideal, não evitando a hiperinsuflação pulmonar e até mesmo pôde colaborar para o aparecimento da mesma. Este estudo também diferenciou-se do experimento realizado por Bianchi et al. ${ }^{(17)}$ que, ao aplicarem CPAP em indivíduos portadores de DPOC, cOm PEEP de $6 \mathrm{CmH}_{2} \mathrm{O}$, em bicicleta ergométrica e intensidade de esforço de $80 \%$ do $\mathrm{VO}_{2}$ máx, observaram aumento da resistência à fadiga e diminuição da sensação de desconforto em membros inferiores, porém, com o nível de fc mantendo-se semelhante ao do grupo controle. 0 ergômetro utilizado e o nível de PEEP aplicado podem ter contribuído para essa constatação.

O presente trabalho pôde concluir que os indivíduos portadores de DPOC, submetidos à atividade física em esteira ergométrica, apresentaram aumento da CVF, de maneira transitória, na comparação pré e pós-atividade física e diminuição da f e da fc quando submetidos à aplicação da CPAP, nas duas situações estudadas, com a mesma intensidade de esforço submáximo. $\mathrm{A} \mathrm{SaO}_{2}$ apresentou maiores valores somente quando se utilizou a CPAP com $\mathrm{FiO}_{2}$ a $30 \%$.

Esses fatos somam-se aos resultados obtidos por outros autores ${ }^{(11-13,17)}$ e diferenciam-se, como já foi discutido, dos que também estudaram os efeitos da VNI em indivíduos portadores de DPOC durante atividade física(12,17).

\section{REFERÊNCIAS}

1. West JB. Fisiologia respiratória moderna. 5a ed. São Paulo: Manole, 1996.

2. Dodd DS, Brancatisano T, Engel LA. Chest wall mechanics during exercise in patients with severe chronic airflow obstruction. Am Rev Respir Dis 1984;129:33-8.
3. Fleury B, Murciano C, Talmo C, Aubier M, Pariente R, Milic-emili J. Work of breathing in patients with chronic obstructive pulmonary disease in acute respiratory failure. Am Rev Respir Dis 1985;131:822-7.

4. Milic-emili J, Chartrand D, Haluska J. Intrinsic Peep in stable COPD patients. Bull Eur Physiopathol Respir 1986;22:415-8.

5. Mcardle WD, Katch FI, Katch VL. Fisiologia do exercício: energia, nutrição e desempenho humano. 4⿳亠丷a ed. Rio de J aneiro: Guanabara, 1998;695.

6. Hyatt RE, Wilcox RE. Extrathoracic airway resistance in man. J Appl Physiol 1961;16:326-30.

7. Marini JJ, Culver BH, Butler J. Mechanical effect of lung distension with positive pressure on cardiac function. Am Rev Respir Dis 1979; $124: 382-6$

8. Kackmareck RM, Petty TL. Historical development of positive endexpiratory pressure (Peep). Respiratory Cares 1988;33:422-33.

9. Clark TJH, Fredmann S, Campbell EJM, Winn RR. The ventilatory capacity of patients with chronic airway obstruction. Clin Sci 1969; 36:307-16.

10. J ones $\mathrm{NL}$, J ones $\mathrm{G}$, Edwards RHT. Exercise tolerance in chronic airway obstruction. Am Rev Respir Dis 1971;103:477-91.

11. O'Donnell DE, Sanii R, Giesbrecht G, Younes M. Effect of continuous positive airway pressure on respiratory sensation in patients with chronic obstructive pulmonary disease during submaximal exercise. Am Rev Respir Dis 1988;138:1185-90.

12. Petrof BJ, Legare M, Goldberg P, Milic-emili J, Gottifried SB. Continuous positive airway pressure reduces inspiratory work of breathing and dyspnea during weaning from mechanical ventilation in severe chronic obstructive pulmonary disease. Am Rev Respir Dis 1990;141: 281-9.

13. Dolmage TE, Goldstein RS. Proportional assist ventilation and exercise tolerance in subjects with COPD. Chest 1997;111:948-54

14. Pereira CAC, Lemle A, Algranti E, J amsen J M, Valença LM, Nery LE, et al. I Consenso Brasileiro sobre Espirometria. J Pneumol 1996;22: 105-64.

15. Karvonen $\mathrm{MJ}$, et al. The effects of training on heart rate. A longitudinal study. Ann Med Exp Biol Finn 1957;35:305.

16. Hoel PG. Estatística elementar. São Paulo: Atlas, 1981;430.

17. Bianchi L, Foglio K, Pagani M, Vitacca M, Rossi A, Ambrosino N. Effects of proportional assist ventilation on exercise tolerance in COPD patients with chronic hypercapnia. Eur Respir J 1988;11:422-7.

18. Fishman AP. Diagnóstico das doenças pulmonares. 2 a ed. São Paulo: Manole, 1992;2:1359-65.

19. Gattinoni L, Pelosi P, Vitale G, Pesenti A, D'Andrea L, Mascheroni D. Body position changes redistribute lung computed tomographic density in patients with acute respiratory failure. Anesthesiology 1991;74:1523.

20. Eschenbacher WL, Sheppard D. Respiratory heat loss is not the sole stimulus for bronchoconstriction induced by isocapnic hyperpnea with dry air. Am Rev Respir Dis 1985;113:131-9.

21. Emmerich JC. Suporte ventilatório: conceitos atuais. 2a ed. Rio de J aneiro: Revinter, 1998;120. 